\title{
INFLUENCE OF THE LOCAL ENVIRONMENT ON THE MAGNETIC PROPERTIES OF THE ORDERED $\mathrm{Fe}_{1-x} \mathrm{Al}_{x}$ ALLOY
}

\author{
J. DeniszczyK* \\ Institute of Physics and Chemistry of Metals, Silesian University \\ Bankowa 12, 40-007 Katowice, Poland
}

The electronic and magnetic properties of the ordered binary $\mathrm{Fe}_{1-x} \mathrm{Al}_{x}$ alloy with the $B 2$ crystal structure were calculated for $\mathrm{Al}$ concentrations $x=0.1875-0.5$ using the supercell structure. The calculations showed that for increasing $\mathrm{Al}$ contents the average magnetization of $\mathrm{Fe}_{1-x} \mathrm{Al}_{x}$ falls down rapidly. Upon lowering the unit cell volume, almost all configurations of compositions with $x \geq 0.25$ show the abrupt change of magnetic state from the high moment magnetic state to the low moment magnetic state with the moment reduction $0.6-0.8 \mu_{\mathrm{B}}$ per atom. The configurations were found for which the low moment magnetic state solutions with the antiferromagnetic alignment of magnetic moments of $\mathrm{Fe}$ atoms at antisite positions are stable.

PACS numbers: 71.15.La, 71.15.Fv, 71.20.Lp, 75.50.Bb, 75.30.-m

The ordered $B 2$-type $\mathrm{Fe}_{1-x} \mathrm{Al}_{x}$ alloys show many anomalous magnetic properties for Al concentrations $x>0.2$. At low Al concentrations $(x<0.2)$ the magnetization decreases following the simple dilution law, at a rate $2.2 \mu_{\mathrm{B}}$. For concentration beyond 20 at.\% $\mathrm{Al}$ the saturation magnetization decreases sharply for increasing Al contents. Arrott and Sato [1] explained this rapid change of the magnetization as a result of antiferromagnetic coupling between iron magnetic moments. The existence of antiferromagnetic alignment of $\mathrm{Fe}$ moments in the ordered $\mathrm{Fe}-\mathrm{Al}$ alloys was also postulated by Shiga and Nakamura [2]. Another commonly used model [3] ascribes the rapid fall of magnetic moment in the $\mathrm{Fe}-\mathrm{Al}$ alloys to the effect of the local environment and neglects the possible antiferromagnetic order of iron moments. Electronic structure calculations of stoichiometric FeAl compound have shown the existence of metastable antiferromagnetic solution lying on the energy scale closely to the ferromagnetic one [4]. Also the cluster calculations of Elzain and Yousif [5] have shown that the specific configuration of $\mathrm{Fe}$ atoms at the nearest neighbor $(\mathrm{nn})$ and next nearest neighbor ( $\mathrm{nnn}$ ) positions can produce the negative magnetic moment at the center Fe atom. Though the alloys were

*e-mail: jdeni@us.edu.pl 
widely studied both experimentally and theoretically, the microscopic picture of the magnetic anomalous behavior of $\mathrm{Fe}-\mathrm{Al}$ ordered alloys is still not clear.

The ordered $B 2$-type $\mathrm{Fe}_{1-x} \mathrm{Al}_{x}$ alloys produced by quenching from high temperatures will be chemically disordered and will contain clusters in which the Fe-sublattice is completely occupied by iron atoms and the Al-lattice is occupied randomly by $\mathrm{Al}$ or $\mathrm{Fe}$ atoms. To study the influence of the local environment on the magnetic properties of the ordered $\mathrm{Fe}_{1-x} \mathrm{Al}_{x}$ the supercell was constructed with translational vectors $(2 a, 0,0),(0,2 a, 0),(0,0,2 a)$ of original $B 2$ lattice, i.e. including 16 atoms ( $8 \mathrm{FeAl}$ formula units). The calculations were performed for six $\mathrm{Al}$ concentrations in the range $x=0.1875-0.5$ corresponding to $0,1,2,3,4$, and 5 $\mathrm{Fe}$ atoms at antisite positions (Fe-AS atoms). In the $2 \times 2 \times 2$ supercells used in the calculations the Fe-AS atoms are all surrounded by the group of 8 crystallographically equivalent iron atoms from the Fe-lattice. Due to the random occupation of the next nearest positions the group of Fe-AS atoms in some configurations splits into different crystallographic classes. In these cases the magnetic moments of the $\mathrm{Fe}-\mathrm{AS}$ atoms belonging to the different classes are allowed to differ in magnitude and alignment.

The calculations were performed with the use of the tight-binding linear muffin-tin orbital (TB-LMTO) method in the atomic sphere approximation (ASA) [6]. Taking into account the results of previous studies [4] it is assumed that all atoms in the supercell have equal and space-filling atomic sphere radii. The exchange-correlation (XC) potential has been used in the form given by von Barth and Hedin [7] with the non-local corrections of Langreth and Mehl [8]. The calculations were performed with the use of the $12 \times 12 \times 12$ mesh in the Brillouin zone which gave the total energy error less than $10^{-4} \mathrm{Ry}$.

The equilibrium lattice parameters obtained for the stoichiometric $B 2 \mathrm{FeAl}$ $(2.871 \AA)$ and the $\mathrm{DO}_{3} \mathrm{Fe}_{3} \mathrm{Al}(5.676 \AA)$ compounds using the same calculation setup as for supercell were smaller than the experimental ones by $1-2 \%$. The ground state of $\mathrm{FeAl}$ was found magnetic $\left(m_{\mathrm{Fe}}=0.72 \mu_{\mathrm{B}}\right)$ with transition to paramagnetic state for lattice constants $a<2.75 \AA$ which is consistent with the results reported earlier by several authors (see e.g. Ref. [9]). The local and bulk magnetic moments calculated for $\mathrm{Fe}_{3} \mathrm{Al}$ are in good agreement with the results of measurements extrapolated to $0 \mathrm{~K}$. Comparison with the data obtained for $\mathrm{FeAl}$ and $\mathrm{Fe}_{3} \mathrm{Al}$ by the same method but with the use of different sets of atomic sphere radii [10] shows that the choice of radii set is of minor importance with respect to the magnetic properties.

For Al concentrations $x \geq 0.25$ almost all examined configurations display the high moment (HM) magnetic state at the upper range and the low moment (LM) magnetic state at the lower range of lattice constants with the abrupt change of magnetic moment in between. Figure 1 is an example of such behavior observed for three different configurations of $\mathrm{Fe}-\mathrm{AS}$ atoms in the $\mathrm{Fe}_{11} \mathrm{Al}_{5}$ supercell (Table summarizes the magnetic results for these configurations). The magnetic state of $\mathrm{Fe}_{13} \mathrm{Al}_{3}$ composition and of the $\mathrm{Fe}_{12} \mathrm{Al}_{4}$ composition with $\mathrm{DO}_{3}$ structure do not show any abrupt changes of magnetic moment when lattice constant is varied. As can be seen from Fig. 1a the configuration (a) of $\mathrm{Fe}_{11} \mathrm{Al}_{5}$ in the lower range of lattice constants is characterized by two magnetic states lying closely on the 
energy scale (the energy difference is $0.6 \mathrm{mRy} \cdot \mathrm{fu}^{-1}$ ). The stable magnetic state is that with one of the Fe-AS magnetic moments antiparallel to supercell magnetic moment (see Table). The same magnetic behavior was found for two configurations of $\mathrm{Fe}_{12} \mathrm{Al}_{4}$ supercell.

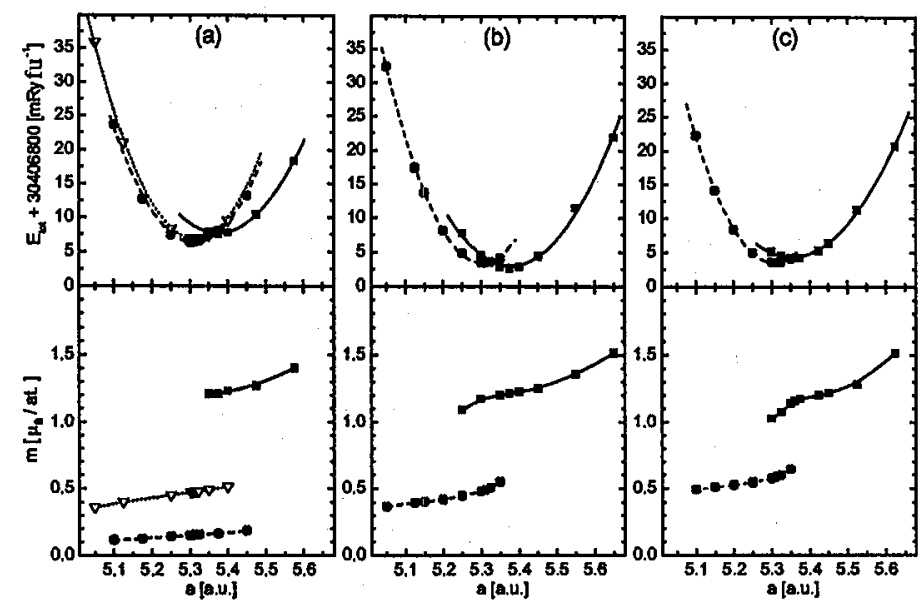

Fig. 1. Total energy per formula unit (upper panels) and total magnetic moment per atom (lower panels) vs. lattice constant for the $\mathrm{Fe}_{11} \mathrm{Al}_{5}$ supercell. Solid, dashed, and dotted lines indicate different magnetic solutions. Parts (a), (b), and (c) represent the different Fe-AS configurations.

\section{TABLE}

Magnetic results for the three configurations and different magnetic state of $\mathrm{Fe}_{11} \mathrm{Al}_{5}$ (in units of $\mu_{\mathrm{B}} /$ at.).

\begin{tabular}{|c|c|c|c|c|c|c|}
\hline \multirow[b]{2}{*}{ Configuration } & \multirow{2}{*}{$\begin{array}{c}\text { Magnetic } \\
\text { state }\end{array}$} & \multicolumn{5}{|c|}{ Magnetic moment } \\
\hline & & $\begin{array}{c}\mathrm{Fe} \\
\text { sublattice }\end{array}$ & & $\begin{array}{l}\mathrm{Fe}-\mathrm{A} \\
\text { atom }\end{array}$ & & Average \\
\hline \multirow{3}{*}{ (a) } & strong & 1.53 & 2.54 & 2.54 & 2.64 & 1.77 \\
\hline & medium & 0.13 & 2.18 & 2.18 & 2.28 & 0.69 \\
\hline & weak & 0.04 & 2.08 & 2.08 & -2.06 & 0.22 \\
\hline \multirow[t]{2}{*}{ (b) } & strong & 1.58 & 2.46 & 2.46 & 2.46 & 1.72 \\
\hline & medium & 0.19 & 2.14 & 2.14 & 2.14 & 0.72 \\
\hline \multirow[t]{2}{*}{ (c) } & strong & 1.48 & 2.37 & 2.58 & 2.30 & 1.69 \\
\hline & medium & 0.37 & 2.27 & 2.12 & 2.21 & 0.86 \\
\hline
\end{tabular}

In the HM magnetic state it was found that when the $\mathrm{Al}$ concentration increases in the range $0.1875-0.3125$ the total magnetic moment per iron atom 
$\left(\bar{m}_{\mathrm{Fe}}\right)$ decreases linearly from $2.2 \mu_{\mathrm{B}}$ to $1.6 \mu_{\mathrm{B}}$ with the rate $R=\Delta \bar{m}_{\mathrm{Fe}} / \Delta x=$ $-3.2 \mu_{\mathrm{B}}$. Further increase in $x$ produces a step-like reduction of $\bar{m}_{\mathrm{Fe}}$ to the value of $1.4 \mu_{\mathrm{B}}$ in $\mathrm{Fe}_{10} \mathrm{Al}_{6}$ and then the smooth decrease in $\bar{m}_{\mathrm{Fe}}$ up to $0.8 \mu_{\mathrm{B}}$ in $\mathrm{Fe}_{8} \mathrm{Al}_{8}(R=$ $\left.4.8 \mu_{\mathrm{B}}\right)$. The magnetic moment of Fe atoms occupying the Fe-sublattice does not disappear for the number of $\mathrm{Al}$ nearest neighbors greater than 4 . Only for two configurations of $\mathrm{Fe}_{10} \mathrm{Al}_{6}$ the magnetization of $\mathrm{Fe}$-lattice almost vanishes. In the LM magnetic phase the local magnetic structure is more complicated. The main conclusion that can be drawn from the calculations is that the sharp decrease in average magnetic moment is mainly due to the rapid reduction of Fe-sublattice magnetization.

Analysis of the values of critical lattice parameters $\left(a_{\mathrm{c}}\right)$ where the magnetic state of $\mathrm{Fe}_{n} \mathrm{Al}_{m}$ structures changes abruptly from $\mathrm{HM}$ to LM magnetic state has proved that the $a_{\mathrm{c}}$ slowly increases with rising $\mathrm{Al}$ contents. The values of $a_{\mathrm{c}}$ obtained for different calculated compositions range from $2.884 \AA$ in $\mathrm{Fe}_{9} \mathrm{Al}_{7}$ to $2.799 \AA$ in $\mathrm{Fe}_{12} \mathrm{Al}_{4}$. The room temperature measurements reported by Shiga and Nakamura [2] reveal that the unit cell parameter of ordered $\mathrm{Fe}_{1-x} \mathrm{Al}_{x}$ alloys for $x=0.25-0.5$ varies in the range $2.885-2.9 \AA$ showing the minimum for concentrations around 30 at.\% Al. Having in mind the calculated magnetic behavior of $\mathrm{Fe}_{n} \mathrm{Al}_{m}$ structures it may be concluded that the rapid fall down of magnetic moment of an $\mathrm{Fe}_{1-x} \mathrm{Al}_{x}$ alloy for concentrations $x$ around 0.3 could be associated with the transition from HM to LM magnetic state forced by the reduction of lattice parameter. Also the lattice dilatation upon the temperature variation could result in the magnetic transition from HM to LM magnetic state observed experimentally in $\mathrm{Fe}_{1-x} \mathrm{Al}_{x}$ for decreasing temperatures [1].

\section{Acknowledgments}

This work was supported in part by the Committee for Scientific Research under contract No. 2 P03B 12914.

\section{References}

[1] A. Arrott, H. Sato, Phys. Rev. 114, 1420, 1427 (1959).

[2] M. Shiga, Y. Nakamura, J. Phys. Soc. Jpn. 40, 1295 (1976).

[3] S.A. Makhlouf, M. Shiga, K. Sumiyama, J. Phys. Soc. Jpn. 60, 3537 (1991).

[4] V.L. Moruzzi, P.M. Marcus, Phys. Rev. B 47, 7878 (1993).

[5] M.E. Elzain, A.A. Yousif, Hyperfine Interact. 94, 1873 (1994).

[6] O.K. Andersen, O. Jepsen, D. Glötzel, in: Highlights of Condensed Matter Theory, Eds. F. Bassani, F. Fumi, M.P. Tosi, North-Holland, Amsterdam 1985, p. 59.

[7] V. von Barth, L. Hedin, J. Phys. C, Solid State Phys. 5, 1629 (1972).

[8] D.C. Langreth, M.J. Mehl, Phys. Rev. B 28, 1809 (1981); C.D. Hu, D.C. Langreth, Phys. Scr. 32, 391 (1985).

[9] B.I. Min, T. Oguchi, H.J.F. Jansen, A.J. Freeman, J. Magn. Magn. Mater. 54-57, 1091 (1986).

[10] J. Deniszczyk, J.E. Frąckowiak, Acta Phys. Pol. A 91, 455 (1997). 